\title{
The "Cone" stoma: stoma creation in the presence of significant bowel dilatation
}

\author{
A. lo Conte ${ }^{1} \cdot$ K. Wasmann ${ }^{1} \cdot$ P. J. Tanis ${ }^{1} \cdot$ C. J. Buskens ${ }^{1} \cdot$ R. Hompes ${ }^{1} \cdot$ W. A. Bemelman ${ }^{1}$
}

Received: 8 December 2018 / Accepted: 31 December 2018 / Published online: 1 March 2019

(c) The Author(s) 2019

\section{Introduction}

The creation of either an ileostomy or colostomy is usually perceived as a quick routine "act"; however, considering the potential impact on the patient's outcome and quality of life, it deserves the utmost attention to detail, particularly if the stoma will be permanent [1]. Stoma-related complications range from 20 to $70 \%$, and span a great variety of problems [2]. Parastomal hernias occur in up 50\% of patients with variable incidence according to stoma type and configuration; $1.8-28.3 \%$ and $0-6.2 \%$ for end and loop ileostomies, and 4-48\% and 0-30.8\% for end and loop colostomies, respectively [3, 4]. Stoma prolapse, defined as a full-thickness protrusion of bowel through the stoma, occurs in $2-11 \%$ of ileostomies and $2 \%$ of colostomies [2]. These are usually both considered late complications, and are associated with older age, obesity, surgical technique, and bowel obstruction. Stoma ischemia/necrosis, retraction, mucocutaneous separation, and parastomal abscess are considered the early complications, and good surgical technique plays a critical role in preventing them. The formation of a "good" stoma is more complex in case of acute bowel obstruction or chronically distended bowel. The dilated bowel requires a large opening in the abdominal wall and results in a wide skin defect and a large mucocutaneous circumference. For any pouching system to fit, a large central opening needs to be cut which reduces the surface for skin adhesion of the pouch baseplate, which inevitably will cause the leaks of stoma content and skin irritation (Fig. 1).

\footnotetext{
R. Hompes

r.hompes@amc.uva.nl

W. A. Bemelman

w.a.bemelman@amc.uva.nl

1 Department of Surgery, Amsterdam UMC, University of Amsterdam, Meibergdreef 9, 1105 AZ Amsterdam, The Netherlands
}

Here, we describe our technique to create a normally sized stoma in patients with a significant bowel dilatation.

\section{Surgical technique}

An elliptical surgical incision is performed at the previously marked site in the right or left lower quadrants of the abdomen according to the type of stoma.

After subcutaneous dissection, the anterior fascia is incised and the rectus abdominis muscle is separated parallel to its fibers to expose the posterior sheath while avoiding the epigastric vessels. The posterior sheath is carefully incised to avoid injury to underlying bowel. The aperture in the abdominal wall should be generally sized to accommodate the stoma without engorgement of the mesentery.

The dilated bowel (Fig. 2) previously transected with a stapler is extracted through an Alexis wound retractor (Applied Medical, Rancho Santo Margarita, CA, USA).

The stapler line is over-sewn using a polydioxanone (PDS, Ethicon) suture, leaving 2-3 cm unaffected (Fig. 2).

The tip of the stapler line (the not over-sewn segment, B) (Figs. 3,4 ) is the only segment finally exteriorized through the skin opening once the wound retractor is released. Using electrocautery, the bowel is cut at the exteriorized tip (Fig. 5).

Full-thickness eversion of the bowel wall is then performed to create a $2 \mathrm{~cm}$ spout and fixation of the stoma to the skin with PDS3/0 stitches. As such, a cone stoma is created with a normal diameter avoiding a very wide and potentially troublesome stoma (Fig. 6). 


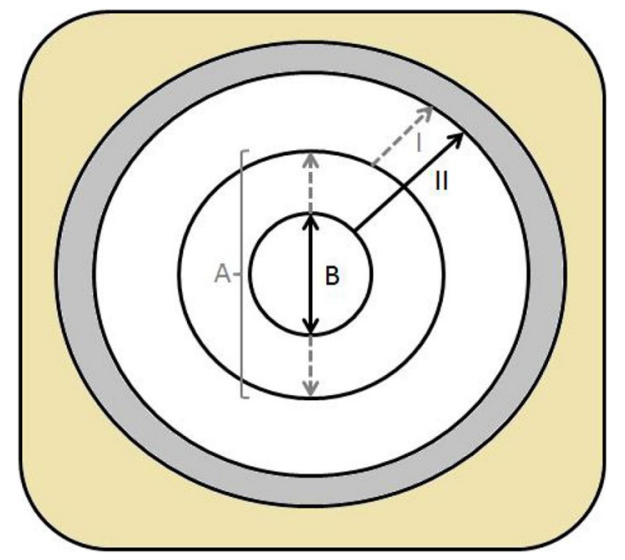

Fig. 1 Ostomy plate. Large stoma: A represents the pouch stoma opening, I: minimal residual adhesive border. "Cone stoma": B is the reduced pouch stoma opening, II: more adhesive margin

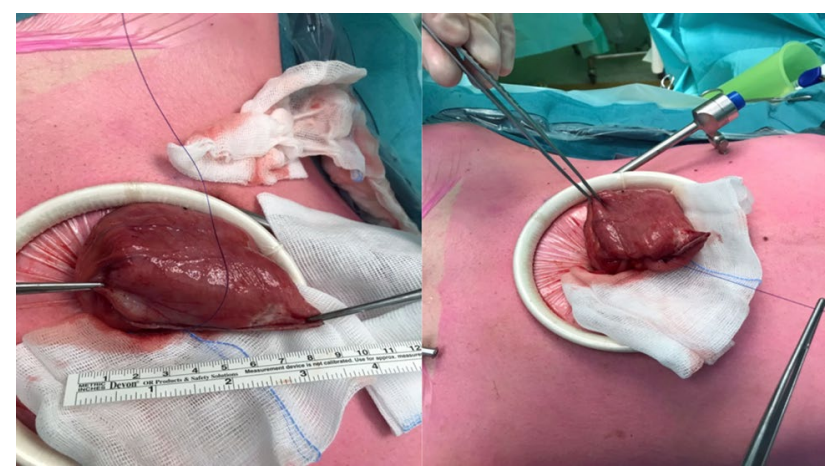

Fig. 2 On the right side: the dilated bowel is extracted from the abdomen. On the left: the stapler line is over-sewn leaving $2-3 \mathrm{~cm}$ unaffected

\section{Conclusions}

This technique is proposed to construct a stoma in cases of chronic bowel obstruction avoiding a large and redundant stoma.

It is a simple and feasible procedure, with better aesthetic and functional results than the classical technique as well as resulting in better patient acceptance.

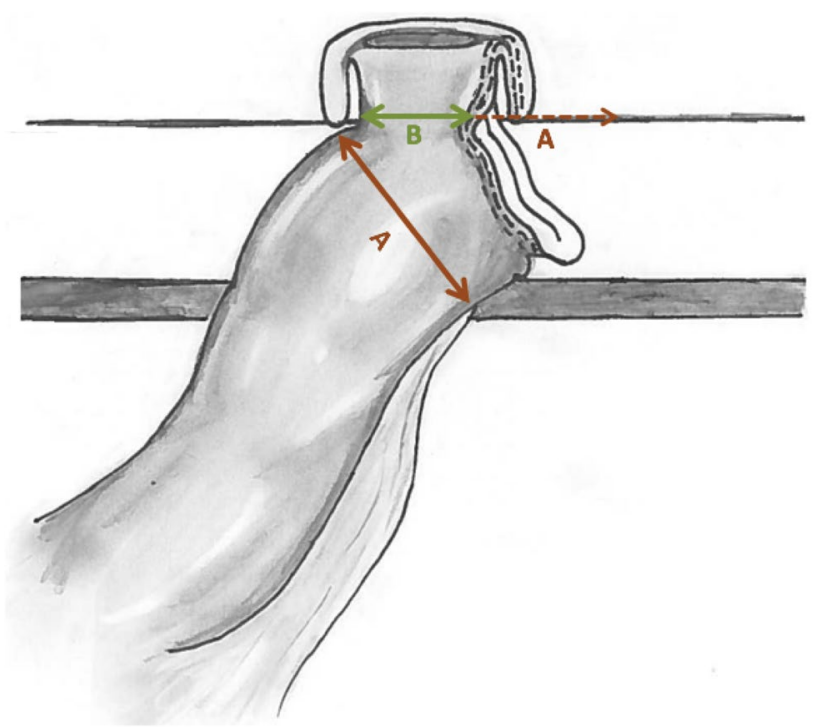

Fig. 3 Continuous line A indicates the diameter of the dilated bowel. Continuous line B is the reduced length of the not over-sewn segment, the only to be exteriorized and used to performed the "Cone" stoma. Dotted line A is the length of the over-sewn bowel segment. Line B+dotted line A represents the eventually stoma size with the classical technique

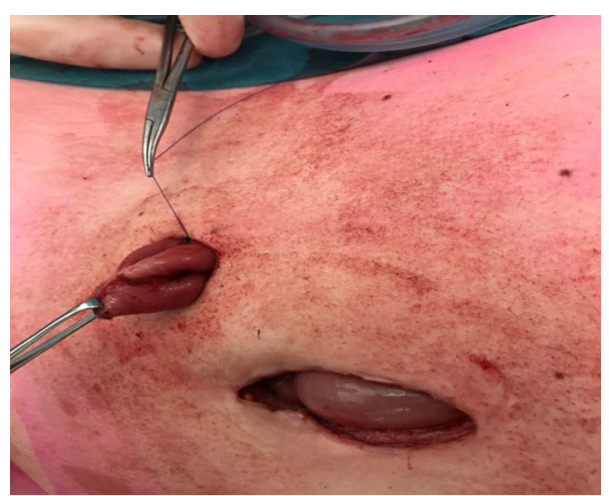

Fig. 4 The not over-sewn segment is the only one to be exteriorized (continuous line $\mathrm{B}$ in the drawing)

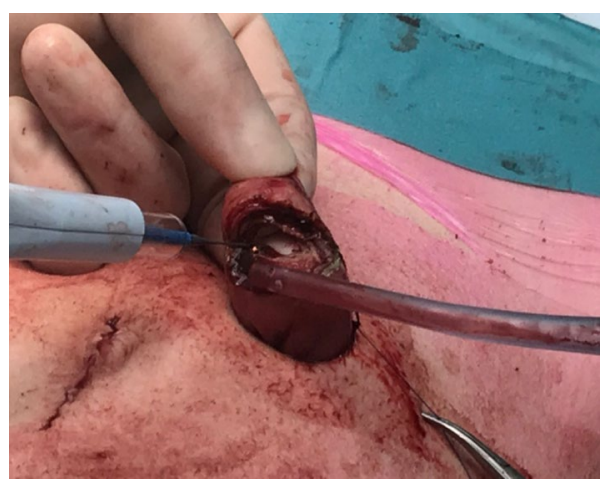

Fig. 5 Incision of the external tip 


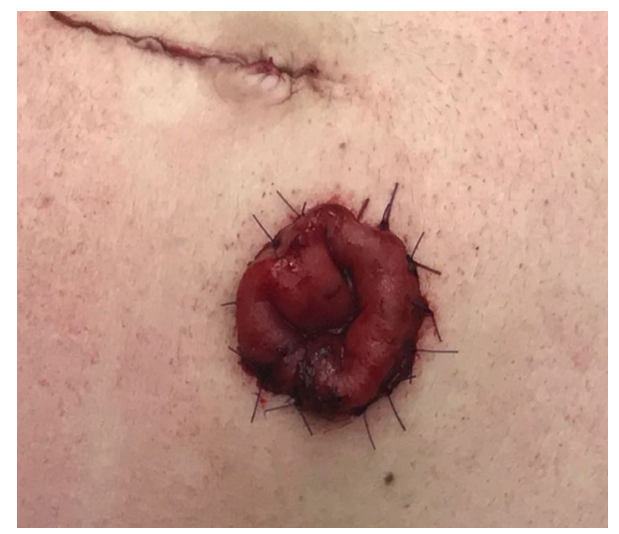

Fig. 6 Stoma is fixed to the skin with PDS 3/0 stitches

\section{Compliance with ethical standards}

Conflict of interest The authors declare that they have no conflict of interest.

Ethical approval The procedure performed in the study were in accordance with the ethical standards of the department and with the 1964 Helsinki declaration and its later amendments or comparable ethical standards.

Informed consent The patient included in the study was formally and individually consented for the procedure.
Open Access This article is distributed under the terms of the Creative Commons Attribution 4.0 International License (http://creativeco mmons.org/licenses/by/4.0/), which permits unrestricted use, distribution, and reproduction in any medium, provided you give appropriate credit to the original author(s) and the source, provide a link to the Creative Commons license, and indicate if changes were made.

\section{References}

1. Scott A, Strong (2016) The difficult stoma: challenges and strategies. Clin Colon Rectal Surg 29(2):152-159. https://doi. org/10.1055/s-0036-1580628

2. Krishnamurty DM, Blatnik J, Mutch M (2017) Stoma complications. Clin Colon Rectal Surg 30:193-200. https://doi. org/10.1055/s-0037-1598160

3. Carne PW, Robertson GM, Frizelle FA (2003) Parastomal hernia. Br J Surg 90(7):784-793. https://doi.org/10.1002/bjs.4220

4. Hannsson BM, Slater NJ, van der Velden AS, Groenewoud HM, Buyne OR, de Hingh IH, Bleichrodt RP (2012) Surgical technique for parastomal hernia repair: a systematic review of the literature. Ann Surg 255(4):685-695. https://doi.org/10.1097/SLA.0b013 e31824b44b1

Publisher's Note Springer Nature remains neutral with regard to jurisdictional claims in published maps and institutional affiliations. 